\title{
Cancer therapy combination: green tea and a phosphodiesterase 5 inhibitor?
}

\author{
Chung S. Yang and Hong Wang
}

Department of Chemical Biology and Center of Cancer Prevention Research, Ernest Mario School of Pharmacy, Rutgers, The State University of New Jersey, Piscataway, New Jersey, USA.

\begin{abstract}
The major constituent of green tea, (-)-epigallocatechin-3-O-gallate (EGCG), has been shown to have cancer-preventive and therapeutic activities. Numerous molecular targets for EGCG have been proposed, but the mechanisms of its anticancer activities are not clearly understood. In this issue of the JCI, Kumazoe et al. report that EGCG activates 67-kDa laminin receptor (67LR), elevates cGMP levels, and induces cancer cell apoptosis. Furthermore, a phosphodiesterase 5 inhibitor, vardenafil, synergizes with EGCG to induce cancer cell death. This is a provocative observation with important implications for cancer therapy. It also raises several issues for further investigation, such as the mechanism by which EGCG specifically activates 67LR.
\end{abstract}

Green tea, a popular beverage made from the leaves of the plant Camellia sinensis, has been studied for the past 25 years for its cancer-preventive and therapeutic activities. Green tea preparations have been shown to inhibit tumorigenesis in animal models for cancers of the lung, oral cavity, esophagus, stomach, small intestine, colon, skin, liver, pancreas, bladder, prostate, and mammary glands (reviewed in ref. 1). Most of the inhibitory activities have been attributed to the major and characteristic tea polyphenol, (-)-epigallocatechin-3-Ogallate (EGCG) (Figure 1). EGCG is an antioxidant with strong binding affinities to biological molecules, but has only limited systemic bioavailability when ingested orally. After consumption of the equivalent of two or three cups of green tea, the peak plasma levels of EGCG are usually $0.2-0.3 \mu \mathrm{M}$. With high oral doses of tea polyphenols (e.g., 800 mg EGCG per day), a peak plasma level as high as $3.5 \mu \mathrm{M}$ has been reported (2). In black tea, the levels of EGCG are only $10 \%-25 \%$ those of green tea because the monomeric tea polyphenols are polymerized to form theaflavins and thearubigins, which have little or no systemic bioavailability. Thus, consumption of green tea is frequently associated with decreased risk for gastrointestinal tract cancers, whereas intake of black tea is rarely associated with such a benefit (3).

Conflict of interest: The authors have declared that no conflict of interest exists.

Citation for this article: J Clin Invest. 2013; 123(2):556-558. doi:10.1172/JCI67589.

\section{Proposed mechanisms of the anticancer actions of EGCG}

Many mechanisms for the anticancer activities of EGCG have been proposed and reviewed $(1,4)$; some are summarized in Figure 1 . EGCG is known to chemically reduce ROS. Its antioxidant activities have been demonstrated in vivo, especially under conditions of increased oxidative stress caused by smoking, chemical carcinogens, and aging. Since ROS accumulation is a hallmark of cancer, this may be a mechanism by which EGCG inhibits carcinogenesis. On the other hand, EGCG can be auto-oxidized to produce ROS, as shown in vitro and in some xenograft tumors (5). Whether EGCGgenerated ROS can induce cancer cell apoptosis clinically remains to be investigated.

The multiple phenolic groups of EGCG can serve as hydrogen bond donors to many biomolecules. For example, EGCG was demonstrated to directly bind to the antiapoptotic B cell CLL/lymphoma 2 protein, resulting in induction of apoptosis (6). Several EGCG binding or target proteins have been identified, including vimentin, IGF-1 receptor (IGF-1R), glucose-regulated protein $78 \mathrm{kDa}$, and peptidyl cis/trans isomerase $(4,7)$. Each of these proteins was shown to be important for the inhibitory activity of EGCG in the cell line used for the study, but the effective EGCG concentrations were much higher than the $K_{d}$ values for the EGCG-protein binding. EGCG has been shown to inhibit the activities of a variety of enzymes. These include some of the MAPKs (leading to inhibition of AP-1), DNA methyltransferases (leading to the demethylation and reactiva- tion of tumor suppressor genes), proteases such as MMP2 and MMP9, dihydrofolate reductase, and GAPDH. In addition, many studies have demonstrated the inhibitory effects of EGCG on membrane receptors, such as IGF-1R, EGFR, and HGFR, by direct binding, inhibiting ligand-receptor binding, or inducing internalization of the receptors into endosomes. Of particular interest is activation of the $67-\mathrm{kDa}$ laminin receptor (67LR), the focus of the current paper by Kumazoe et al. (8). Laminin-1 is the major glycoprotein of the basement membrane and is recognized for its roles in cell adhesion, differentiation, proliferation, and migration as well as tumor growth and metastasis (9); the mechanism of $67 \mathrm{LR}$ signaling is not well understood.

\section{Synergistic action between EGCG} and vardenafil in inducing apoptosis Binding of EGCG to the $67 \mathrm{LR}\left(K_{d}, 0.04 \mu \mathrm{M}\right)$ was first observed by Tachibana et al. using a surface plasma resonance assay (10). Expression of the metastasis-associated $67 \mathrm{LR}$ increases the responsiveness of MCF-7 cells to low micromolar concentrations of EGCG (10). Furthermore, silencing of 67LR abrogates EGCG-induced apoptosis in multiple myeloma (MM) cells (11). Recent studies by this group elucidated the critical role of 67LR in mediating antiinflammatory action of EGCG on macrophages (12). The present work by Kumazoe et al. showed that the activation of 67LR by EGCG in primary MM cells and in MM cell lines (U266, ARH-77, and RPMI 8226) resulted in elevated levels of cGMP, which initiated apoptosis through the activation of PKC $\delta$ and acid sphingomyelinase in a novel death pathway (8). However, EGCG alone was not a very effective in killing MM U266 cells ( $\mathrm{IC}_{50}, 23.2 \mu \mathrm{M}$ ), because these cells overexpressed phosphodiesterase 5 (PDE5), a negative regulator of cGMP. When a PDE5-selective inhibitor, vardenafil, was also added to cultured cells, it synergized with EGCG to reduce the $\mathrm{IC}_{50}$ of EGCG to $1.4 \mu \mathrm{M}$. The authors also investigated the synergy between EGCG and 


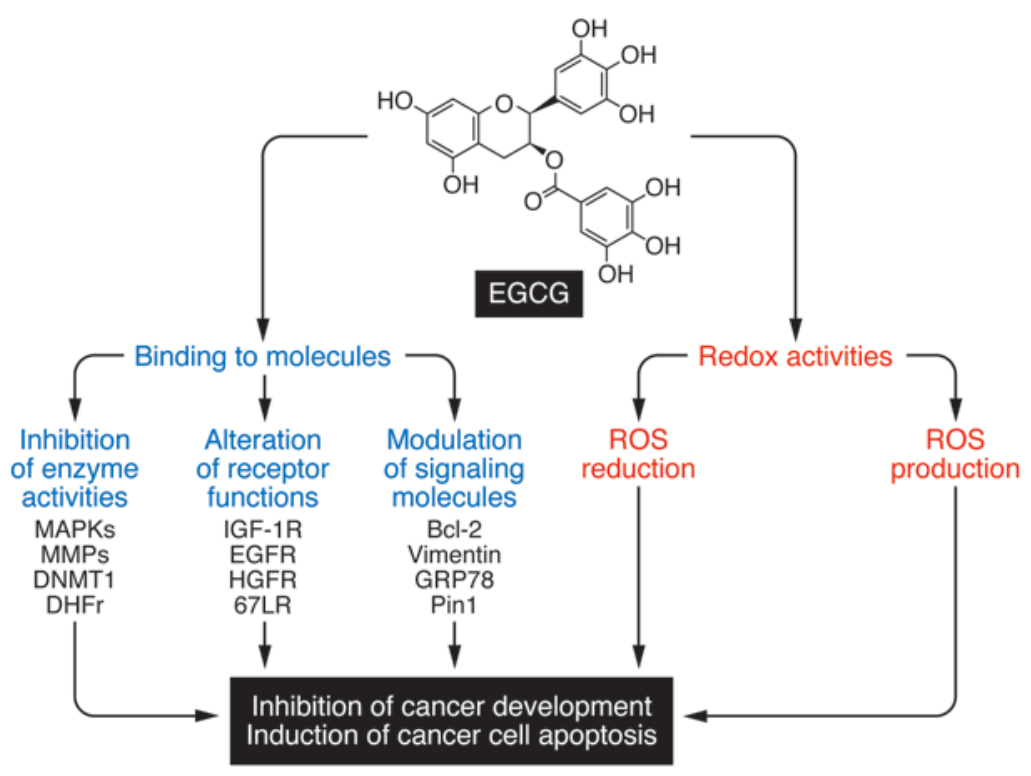

Figure 1

Proposed mechanisms of anticancer action of EGCG. The mechanism by which EGCG exerts its antitumor actions is unknown, but may involve one or more of the actions illustrated. EGCG can act as an antioxidant, reducing ROS and inhibiting cancer development, and paradoxically may promote the production of ROS in cancer cells and induce apoptosis. EGCG is also known to bind and modulate the activities of enzymes, receptors, and signaling molecules that affect cell growth and proliferation. Activation of $67 \mathrm{LR}$ by EGCG and inhibition of PDE5 activity by its inhibitors synergistically induce cancer cell apoptosis (8). DNMT1, DNA, methyltransferase 1; DHFr, dihydrofolate reductase; HGFR, HGF receptor; Bcl-2, B cell CLL/lymphoma 2; GRP78, glucose-regulated protein $78 \mathrm{kDa}$; Pin1, peptidyl cis/trans isomerase.

other PDE5-selective inhibitors: zaprinast, methoxyquinazoline (MQZ), and sildenafil (Viagra). The authors used shRNA, neutralizing antibodies, and enzyme inhibitors to demonstrate the involvement of these key receptors and enzymes in the synergistic action. This impressive synergism was also shown in MM MPC-11 cells and breast cancer MDA-MB-231 cells in a xenograft model as well as in vitro in some gastric, pancreatic, and prostate cancer cell lines. Overexpression of both 67LR and PDE5 was observed in these cell lines (8).

\section{Clinical implications and open questions}

Although EGCG has previously been shown to induce apoptosis of a variety of cancer cell lines (4), its clinical application is limited by low bioavailability. When high doses of tea polyphenols (e.g., 1-2 g EGCG, twice daily) were used for the treatment of chronic lymphocytic leukemia (CLL), hepatotoxicity was observed in some patients (13). The novel approach of combining EGCG with vardenafil to selectively inhibit PDE5 significantly decreased the lethal dose of EGCG for cancer cells that overexpressed both 67LR and PDE5 (8), reducing the chances of side effects of EGCG. The vardenafil dose used in the xenograft experiment ( $5 \mathrm{mg} / \mathrm{kg}$ every other day; ref. 8) was close to the clinical dose range for patients with erectile dysfunction, calculated based on body surface area (14), even though the vardenafil concentration used in cell culture $(5 \mu \mathrm{M})$ was much higher than the peak plasma levels (15 nM) observed in patients after taking $10 \mathrm{mg}$ vardenafil (15). More studies on the dose-response relationship of the synergistic action are needed.

In addition to MM, overexpression of both 67LR and PDE5 was also observed in some gastric, pancreatic, prostate, and breast cancer samples (8). EGCG, a principal constituent of green tea, and PDE5selective inhibitors, commonly used to treat erectile dysfunction, are readily available, and the proposed combination approach may have broad applications. Whether this therapeutic combination can replace established modalities remains to be determined. Nevertheless, the combination of these two agents might be useful after tumor resection to kill the remaining cancer cells and prevent recurrence. Many prostate cancer patients are taking PDE5-selective inhibitors after prostatectomy to treat erectile dysfunction. If their cancer samples overexpress 67LR and PDE5, it would be interesting to determine whether taking an EGCG supplement or a green tea beverage would prevent prostate cancer recurrence.

Because the 67LR-death signaling concept (8) is novel, additional confirmatory research from different laboratories is needed to further substantiate this pathway and answer some fundamental questions. For example, how does EGCG activate 67LR? Previous publications by the Tachibana group reported the binding of EGCG to 67LR (10) and the induction of membrane lipid raft clustering by EGCG in MM cells (16). Although the binding between EGCG and 67LR could be a specific action, alteration of cell membrane structure by EGCG may be a nonspecific event that requires a large amount of EGCG to affect all types of cells, which is unlikely to occur in vivo. What is the relative importance of the different actions proposed for EGCG (Figure 1)? This may depend upon context, and the actions triggered by lower concentrations of EGCG are likely to be the most relevant in vivo. The fact that combination treatment with vardenafil decreased the effective concentration of EGCG to 1.4 $\mu \mathrm{M}(8)$ makes this therapeutic approach more attractive. Nevertheless, the relative importance of these proposed mechanisms still needs to be further investigated.

In clinical situations, a combination of EGCG and vardenafil may kill cancer cells expressing 67LR and PDE5. However, there may also be cells with lower levels of 67LR that can be activated by EGCG. Since 67LR is known to play vital roles in cancer progression, metastasis, invasion, and drug resistance $(9,17)$, whether the activation of 67LR by EGCG promotes cancer progression will need to be investigated.

\section{Acknowledgments}

We thank Kenneth Reuhl and Jayson X. Chen for helpful suggestions. This work was supported by NIH grants CA120915, CA122474, and CA133021.

Address correspondence to: Chung S. Yang, Department of Chemical Biology, Ernest Mario School of Pharmacy, Rutgers, State University of New Jersey, 164 Frelinghuysen Road, Piscataway, New Jersey 088548020, USA. Phone: 732.445.5360; Fax: 732.445.0687; E-mail: csyang@pharmacy. rutgers.edu. 
1. Yang CS, Wang X, Lu G, Picinich SC. Cancer prevention by tea: animal studies, molecular mechanisms and human relevance. Nat Rev Cancer. 2009;9(6):429-439.

2. Chow HH, Hakim IA. Pharmacokinetic and chemoprevention studies on tea in humans. Pharmacol Res. 2011;64(2):105-112.

3. Yuan JM, Sun C, Butler LM. Tea and cancer prevention: epidemiological studies. Pharmacol Res. 2011;64(2):123-135.

4. Yang CS, Wang H. Mechanistic issues concerning cancer prevention by tea catechins. Mol Nutr Food Res. 2011;55(6):819-831.

5. Li GX, et al. Pro-oxidative activities and doseresponse relationship of (-)-epigallocatechin-3-gallate in the inhibition of lung cancer cell growth: a comparative study in vivo and in vitro. Carcinogenesis. 2010;31(5):902-910.

6. Leone $\mathrm{M}$, et al. Cancer prevention by tea polyphenols is linked to their direct inhibition of antiapoptotic Bcl-2-family proteins. Cancer Res.
2003;63(23):8118-8121.

7. Urusova DV, et al. Epigallocatechin-gallate suppresses tumorigenesis by directly targeting Pin 1 . Cancer Prev Res. 2011;4:1366-1377.

8. Kumazoe M, et al. 67-kDa laminin receptor increases cGMP to induce cancer-selective apoptosis. JClin Invest. 2013;123(2):787-799.

9. Nelson J, et al. The $67 \mathrm{kDa}$ laminin receptor: structure, function and role in disease. Biosci Rep. 2008;28(1):33-48.

10. Tachibana H, Koga K, Fujimura Y, Yamada K. A receptor for green tea polyphenol EGCG. Nat Struct Mol Biol. 2004;11(4):380-381.

11. Umeda D, Yano S, Yamada K, Tachibana H. Green tea polyphenol epigallocatechin-3-gallate signaling pathway through $67-\mathrm{kDa}$ laminin receptor. J Biol Chem. 2008;283(6):3050-3058.

12. Hong Byun E, et al. TLR4 signaling inhibitory pathway induced by green tea polyphenol epigallocatechin-3-gallate through $67-\mathrm{kDa}$ laminin receptor. JImmunol. 2010;185(1):33-45.
13. Shanafelt TD, et al. Phase 2 trial of daily, oral polyphenon $\mathrm{E}$ in patients with asymptomatic, Rai stage 0 to II chronic lymphocytic leukemia. Cancer. 2013;119(2):363-370.

14. Reagan-Shaw S, Nihal M, Ahmad N. Dose translation from animal to human studies revisited. FASEB J. 2008;22(3):659-661.

15. Klotz T, et al. Vardenafil increases penile rigidity and tumescence in erectile dysfunction patients: a RigiScan and pharmacokinetic study. World J Urol. 2001;19(1):32-39

16. Tsukamoto S, et al. Green tea polyphenol EGCG induces lipid-raft clustering and apoptotic cell death by activating protein kinase Cdelta and acid sphingomyelinase through a $67 \mathrm{kDa}$ laminin receptor in multiple myeloma cells. Biochem J. 2012;443(2):525-534.

17. Scheiman J, Tseng JC, Zheng Y, Meruelo D. Multiple functions of the 37/67-kd laminin receptor make it a suitable target for novel cancer gene therapy. Mol Ther. 2010;18(1):63-74.

\title{
Why stress is BAD for cancer patients
}

\section{Archana S. Nagaraja, ${ }^{1}$ Guillermo N. Armaiz-Pena, ${ }^{1}$ Susan K. Lutgendorf, ${ }^{2}$ and Anil K. Sood ${ }^{1,3,4}$}

1Department of Gynecologic Oncology and Reproductive Medicine, The University of Texas MD Anderson Cancer Center, Houston, Texas, USA

2Department of Psychology, Department of Urology, and Department of Obstetrics and Gynecology, University of lowa, lowa City, Iowa, USA. ${ }^{3}$ Department of Cancer Biology and ${ }^{4}$ Center for RNA Interference and Noncoding RNA,

The University of Texas MD Anderson Cancer Center, Houston, Texas, USA.

\begin{abstract}
Behavioral stress is known to promote tumor progression in experimental models, but the role of behavioral stress in cancer initiation is less clear. In this issue, Hassan et al. focus on the signaling and biological effects induced by stress hormones that lead to tumor cell evasion from apoptosis, resulting in prostate cancer progression.
\end{abstract}

\section{Behavioral stress and cancer}

A stressor is often defined as a stimulus that is capable of activating the hypothalamic-pituitary-adrenal (HPA) axis and/ or the sympathetic nervous system (SNS). HPA activation induces hypothalamic production of neurohormones, such as corticotropin-releasing hormone and vasopressin. This, in turn, results in secretion of the adrenocorticotropic hormone from the pituitary and subsequent release of glucocorticoids from the adrenal cortex. SNS activation results in the release of epinephrine and norepinephrine from sympathetic neurons and adrenal medulla. At a broad level, behavioral stress can be considered as acute or chronic. While acute stress may be adaptive for preservation of the organism and can have beneficial effects (e.g., enhanced immune response), chronic stress can lead to disease states such as car-

Conflict of interest: The authors have declared that no conflict of interest exists.

Citation for this article: J Clin Invest. 2013; 123(2):558-560. doi:10.1172/JCI67887. diovascular and metabolic diseases. Moreover, a growing number of studies have uncovered major roles for chronic stress in cancer progression (1).

Catecholamine-dependent signaling is known to promote several protumoral processes that collectively result in increased tumor progression. For example, chronic stress results in increased epinephrine and norepinephrine, whereas dopamine levels are reduced. This catecholamine shift leads to a microenvironment that is conducive to increased tumor growth and progression in experimental models of disease (2). Specifically, elevated norepinephrine levels have been associated with increased angiogenesis, invasion, and protection from anoikis (2-5). Adrenergic activation has been implicated as the key mediator of these effects by modulating several growth factors (e.g., VEGF, IL-6, IL-8, matrix metalloproteinases, and FAK) in multiple cancers. Upon $\beta$-adrenergic receptor (ADRB) activation, increased cAMP-PKA activity is frequently noted as an intracellular mediator of the stress response.
Chronic stress plays a significant role in cancer progression, and decreased cancer incidence is observed among patients who take beta blockers for the treatment of other diseases (6). Cancer diagnosis and associated treatment can potentially elevate a patient's stress levels, whereas social support has been associated with increased patient survival (7). Recent findings regarding the role of stress hormones in chemoresistance, metastasis, cancer relapse, and surgical recovery have moved the field forward, but the molecular mechanisms underlying these effects are not fully understood $(6,8)$. In this issue of the JCI, Hassan et al. have used a variety of experimental models of prostate cancer in an effort to demonstrate the underlying mechanisms by which behavioral stress promotes tumor growth and to provide the basis to support pharmacological and behavioral interventions for prostate cancer patients (9).

\section{Behavioral stress inhibits tumor cell apoptosis}

The acquired ability of tumor cells to evade apoptosis is a classic hallmark of cancer (10). In advanced prostate cancer, activating antiapoptotic signaling is believed to be an important factor in chemoresistance and androgen-independent tumor growth (11). In the study by Hassan et al., epinephrine 